\section{Acknowledgments}

I apologize to authors whose work could not be cited due to space limitations. Work in W.S. Pear's laboratory is supported by grants from the NIH and the Leukemia and Lymphoma Society.

Address correspondence to: Warren S. Pear, Department of Pathology and Laboratory Medicine, Abramson Family Cancer Research Institute, University of Pennsylvania, Philadelphia, Pennsylvania 19104-6160, USA. Phone: (215) 573-7764; Fax: (215) 5736875; E-mail:wpear@mail.med.upenn.edu.

1. Curatolo P, Bombardieri R, Jozwiak S. Tuberous sclerosis. Lancet. 2008;372(9639):657-668.

2. Crino PB, Nathanson KL, Henske EP. The tuberous sclerosis complex. $N$ Engl J Med. 2006;355(13):1345-1356.
3. Inoki K, Guan KL. Tuberous sclerosis complex, implication from a rare genetic disease to common cancer treatment. Hum Mol Genet. 2009;18(R1):R94-R100.

4. Huang J, Manning BD. The TSC1-TSC2 complex: a molecular switchboard controlling cell growth. Biochem J. 2008;412(2):179-190.

5. Sarbassov DD, Ali SM, Sabatini DM. Growing roles for the mTOR pathway. Curr Opin Cell Biol. 2005;17(6):596-603.

6. Guertin DA, Sabatini DM. Defining the role of mTOR in cancer. Cancer Cell. 2007;12(1):9-22.

7. Plas DR, Thomas G. Tubers and tumors: rapamycin therapy for benign and malignant tumors. Curr Opin Cell Biol. 2009;21(2):230-236.

8. Bissler JJ, et al. Sirolimus for angiomyolipoma in tuberous sclerosis complex or lymphangioleiomyomatosis. NEngl J Med. 2008;358(2):140-151.

9. Karbowniczek M, et al. The evolutionarily conserved TSC/Rheb pathway activates Notch in tuberous sclerosis complex and Drosophila external sensory organ development. J Clin Invest. 2010;120(1):93-102.

10. Ma J, et al. Mammalian target of rapamycin regulates murine and human cell differentiation through STAT3/p63/Jagged/Notch cascade. J Clin Invest. 2010;120(1):103-114.

11. Karbowniczek M, Yu J, Henske EP. Renal angiomyolipomas from patients with sporadic lymphangiomyomatosis contain both neoplastic and non-neoplastic vascular structures. Am J Pathol. 2003;162(2):491-500.

12. Artavanis-Tsakonas S, Rand MD, Lake RJ. Notch signaling: cell fate control and signal integration in development. Science. 1999;284(5415):770-776.

13. Posakony JW. Nature versus nurture: asymmetric cell divisions in Drosophila bristle development. Cell. 1994;76(3):415-418.

14. Potter CJ, Pedraza LG, Huang H, Xu T. The tuberous sclerosis complex (TSC) pathway and mechanism of size control. Biochem Soc Trans. 2003;31(Pt 3):584-586.

15. Roegiers F, Jan YN. Asymmetric cell division. Curr Opin Cell Biol. 2004;16(2):195-205.

16. Palomero T, Ferrando A. Oncogenic NOTCH1 control of MYC and PI3K: challenges and opportunities for anti-NOTCH1 therapy in T-cell acute lymphoblastic leukemias and lymphomas. Clin Cancer Res. 2008;14(17):5314-5317.

\title{
Oxidant stress derails the cardiac connexon connection
}

\author{
Gordon F. Tomaselli
}

Department of Medicine, Johns Hopkins University School of Medicine, Baltimore, Maryland, USA.

Connexin $43(\mathrm{Cx} 43)$ is the major protein component of gap junctions that electrically couple cardiomyocytes at the intercalated disc. Oxidant stress, reduced $C_{x} 43$ expression, and altered subcellular localization are present in many forms of structural heart disease. These changes in $\mathrm{Cx} 43$ lead to alterations in electrical conduction in the ventricle and predispose to lethal cardiac arrhythmias. In their study in this issue of the JCI, Smyth et al. tested the hypothesis that oxidant stress perturbs connexon forward trafficking along microtubules to gap junctions (see the related article beginning on page 266). Failing human ventricular myocardium exhibited a reduction in $\mathrm{Cx} 43$ and the microtubule-capping protein EB1 at intercalated discs. Oxidant stress in the adult mouse heart reduced $\mathrm{N}$-cadherin, EB1, and $\mathrm{Cx} 43$ colocalization. In HeLa cells and neonatal mouse ventricular myocytes, peroxide exposure displaced EB1 from the plus ends of microtubules and altered microtubule dynamics. Mutational disruption of the EB1-tubulin interaction mimicked the effects of oxidant stress, including a reduction in surface $\mathrm{Cx} 43$ expression. These data provide important new molecular insights into the regulation of $\mathrm{Cx} 43$ at gap junctions and may identify targets for preservation of cellular coupling in the diseased heart.

Rapid propagation of electrical impulses in excitable tissue is essential to processes as diverse as cognition, movement, and the genesis of the heartbeat. Central to rapid conduction in the heart and other organs are gap junctions. Gap junctions are low-resistance conduits between cells,

Conflict of interest: The author has received a grant from Boston Scientific Inc.

Citation for this article: J. Clin. Invest. 120:87-89 (2010). doi:10.1172/JCI41780. comprised of proteins called connexins. In the heart, connexins are key mediators of electrical conduction and are thus central to excitation and contraction. Connexins hexamerize to form connexons or hemichannels in the membranes of apposing cells that dock head-to-head to form intact gap junction channels (Figure 1).

The major connexin of working ventricular myocardium is connexin 43 (Cx43). Cx43 is richly endowed with protein interaction domains and sites of phosphorylation that contribute to regulation of the functional expression of gap junction channels. The carboxyl terminus contains a PDZ-binding domain, multiple consensus serine and tyrosine phosphorylation sites, and binding sites for tubulins. Post-translational modifications and protein-protein interactions are thought to be important for proper formation and localization of clusters of gap junctions into so-called "plaques," although Cx43 with a truncated carboxyl terminus forms working gap junction channels (1).

\section{Structural complexity of intercalated discs}

The cardiomyocyte is a complex and highly structured cell. In normal myocardium, gap junction channels are prominently located at intercalated discs positioned at cell ends, mediating electrical propagation that is preferentially in the direction of the long axis of the cell (Figure 1). A remarkable feature of gap junctions and other ion channels is their extremely short half-life (approximately 1-3 hours) $(2,3)$. The short half-life of gap junction channels is particularly surprising given the transmembrane topology and the complex structure of the intercalated disc that houses connexin-containing gap junctional plaques and implies highly coordinated and tightly regulated trafficking mecha- 

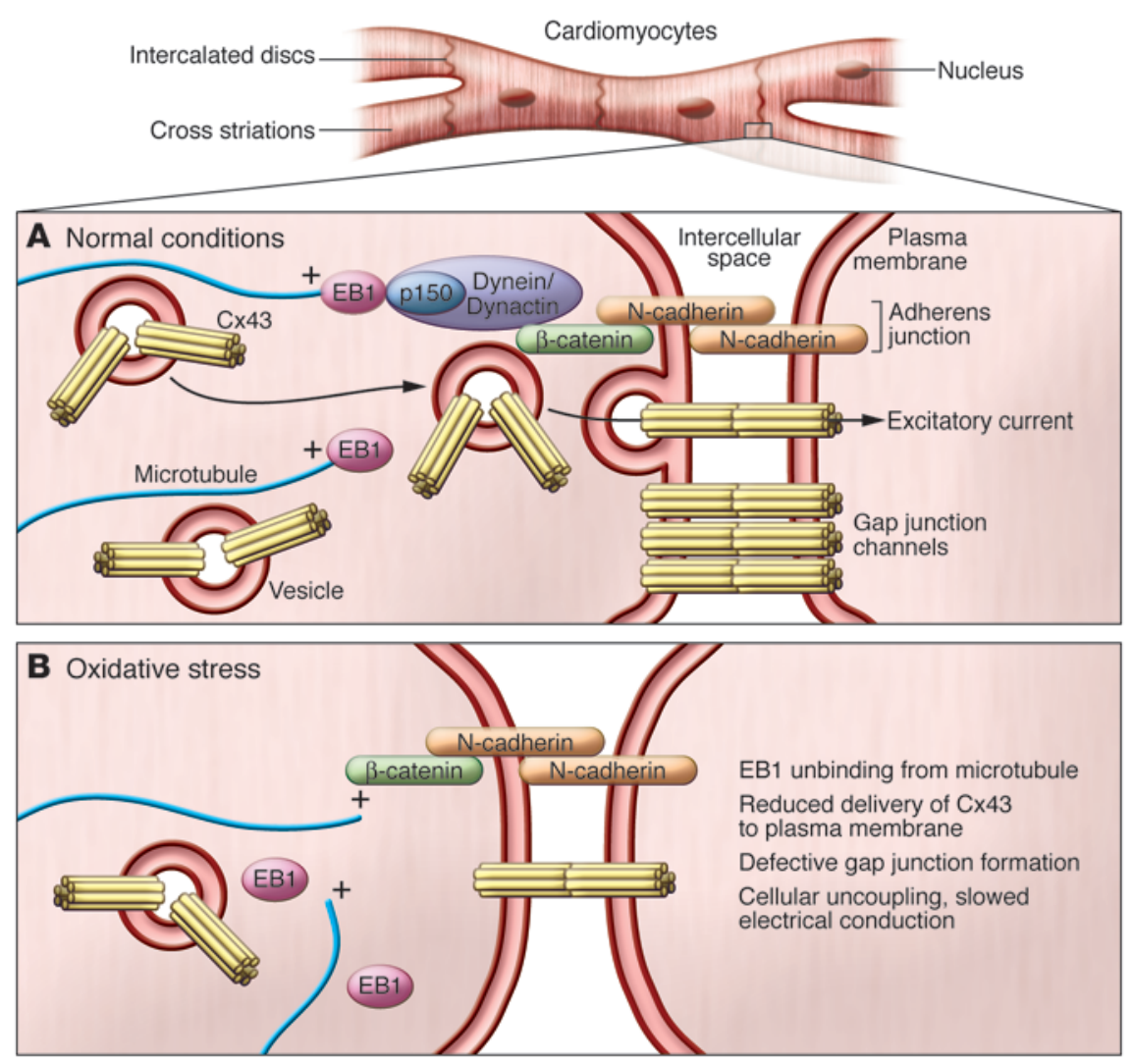

Figure 1

Structure of the cardiac intercalated disc. (A) A schematic of the intercalated disc under normal conditions shows normal forward trafficking along microtubules of Cx43-containing connexons to the adherens junction for incorporation into gap junction plaques between cardiomyocytes. Connexins mediate electrical conduction between cells and are thus central to cardiomyocyte excitation and contraction. (B) In this issue of the $J C l$, Smyth et al. (9) show that in the setting of oxidative stress, the microtubule-capping protein EB1 dissociates from the microtubule plus end, impeding connexon trafficking to the adherens junction and reducing the generation of gap junction channels. This results in cellular uncoupling and slowed electrical conduction in the ventricle and may predispose to lethal cardiac arrhythmias. Adapted with permission from Cell (10) and ref. 9.

nisms both to and from the cell membrane. The structure of the intercalated disc itself is heterogeneous, being comprised of two types of cell-cell junctions in addition to gap junctions: adherens junctions and desmosomes (Figure 1). Adherens junctions transmit force from cell to cell, and desmosomes constitute the sites of desmin attachment. The spatial relationships of these specialized junctions within the intercalated disc provide for adjacencies of the structures but are not immutable. Gap junction plaques are semi-crystalline arrays ranging from tens to thousands of gap junction channels. Viewed en face, gap junction plaques have a characteristic appearance, with large plaques at the perimeter of the gap junction and small plaques in central interplicate regions (for a review, see ref. 4). The question of how this structural arrangement comes about in development, heart (3). Reduced Cx43 expression and altered subcellular localization contribute to changes in electrical conduction in the heart that are proximate causes of potentially lethal ventricular arrhythmias.

Connexins are co-translationally inserted into the ER membrane for transport through the secretory pathway. In order to form hemichannels, connexins must oligomerize into hexamers; in the case of $\mathrm{Cx} 43$, oligomerization takes place in the transGolgi network (5). The oligomerization of connexins is essential for normal functional expression, particularly subsequent transport to sites in the plasma membrane.

Connexins are transported through the Golgi and packaged into transport vesicles in the trans-Golgi network for delivery to the plasma membrane. The forward trafficking involves $\mathrm{Cx} 43$ binding directly to microtubules $(6,7)$ (Figure 1). However, pharmacological disruption of microtubules reduces the efficiency of $\mathrm{Cx} 43$ delivery to the plasma membrane but does not inhibit the process altogether (8). The article published by Smyth, Shaw, and colleagues in this issue of the JCI (9) and previous work from the same group (10) further define the molecular machinery involved in $\mathrm{Cx} 43$ forward trafficking to the intercalated disc. Gap junctions are known to form in close proximity to N-cadherin-containing adherens junctions, and adherens junctions presage the development of electrical gap junctions in juvenile cardiac tissues (11). In the absence of $\mathrm{N}$-cadherin, gap junctions do not form in the mammalian ventricle. Shaw and colleagues previously examined the role of microtubules and adherens junctions in the trafficking of $\mathrm{Cx} 43$ to the plasma membrane in a vital microscopy study of gap junction formation in HeLa cells. They proposed a model of microtubule-based targeted delivery of $\mathrm{Cx} 43$ directly to gap junction plaques that involves the interaction of the dimeric microtubule plus end-capping protein, EB1. Using siRNA methods, they showed that EB1 interaction with its binding partner p150(Glued) of the dynein/dynactin complex tethers microtubules at the adherens junction by way of interaction with $\beta$-catenin, which interacts with N-cadherin (10) (Figure 1). This model contrasts with another prevailing model of connexin delivery to the plasma membrane, which proposes that connexons are delivered to regions of un-apposed plasma membrane, where they are free to diffuse in the plane of the membrane, coalesce with the edges of existing gap junctions, and subsequently dock with hemichannels in apposing cells 
to form new gap junction channels (6). The two models are not mutually exclusive, and it is tempting to speculate that the relative roles of such forward trafficking may change in pathological conditions, contributing to aberrant localization of $\mathrm{Cx} 43$ gap junction channels.

\section{Forward trafficking and oxidant stress}

The present work (9) extends previous findings and has implications for possible mechanisms of gap junction remodeling in heart disease. Oxidant stress is present in many forms of heart disease and, given the rapid kinetics of $\mathrm{Cx} 43$ turnover, altered trafficking is likely to affect gap junction channel formation. Smyth et al. hypothesized that oxidant stress perturbs connexon forward tracking (9). In human ventricular myocardium from end-stage ischemic myopathic hearts, the authors demonstrated a reduction in $\mathrm{Cx} 43$ and $\mathrm{EB} 1$ at intercalated discs. Oxidant stress induced by ischemia-reperfusion and peroxide perfusion of adult mouse heart similarly reduced $\mathrm{N}$-cadherin and $\mathrm{Cx} 43$ colocalization as well as co-immunoprecipation of $\mathrm{Cx} 43$ and $\mathrm{EB} 1$ by $\mathrm{N}$-cadherin. In HeLa cells and neonatal mouse ventricular myocytes, peroxide exposure displaced EB1 from the plus ends of microtubules, altered microtubule dynamics, and inhibited microtubule approach toward the cell membrane. A mutation in EB1 that disrupted the EB1tubulin interaction produced a phenocopy of oxidant stress, including a reduction in surface $\mathrm{Cx} 43$ expression in the heterologous expression system. In zebrafish, colocalization of a labeled connexin with $\mathrm{N}$-cadherin was reduced with oxidant stress, as was cellular coupling and conduction velocity in the myocardium. Although the mechanism of forward trafficking of labeled Cx48.5 in zebrafish is not defined, these experiments provide a nice proof of concept for relevance in the intact heart.

The study by Smyth et al. (9) makes a compelling case for a defect in forward Cx43 trafficking in the presence of oxidant stress and that EB1 unbinding from microtubules is involved in the faulty trafficking (Figure 1). As with all provocative work, a number of questions are raised by these data. It is a certainty that the changes in $\mathrm{Cx} 43$ and gap junction disposition in the setting of ischemia reperfusion or other interventions that induce oxidant stress are multifactorial. Changes in the rate and site of delivery are important, but stabilization of gap junction channels in the plaque, as well as altered rates of internaliza- tion and degradation, are likely to be equally important. The role of other interacting proteins such as zona occludens $1(\mathrm{ZO}-1)$ in this process continues to be debated (12-15). A prominent feature in diseased myocardium is lateralization of immunoreactive $\mathrm{Cx} 43$. The mechanism by which this change in protein distribution occurs is an area of active investigation that may or may not be related to aberrant delivery of $\mathrm{Cx} 43$ to the plasma membrane. The mechanism of the oxidantinduced disruption in the EB1-tubulin interaction, central to defective delivery of $\mathrm{Cx} 43$ to the intercalated disk, is not revealed by these data and is likely to be important in developing therapeutic targets that restore normal trafficking of $\mathrm{Cx} 43$.

\section{Connexin trafficking in heart disease}

Oxidant stress in the diseased heart varies over time and thus only intermittently interferes with forward trafficking of $\mathrm{Cx} 43$. The rate and extent of reversibility of the effects of oxidant stress on forward trafficking are likely to affect the degree of gap junction remodeling. Furthermore, the changes in forward trafficking need to be considered in the context of the alterations in network properties of the myocardium that accompany structural heart disease and oxidant stress. It is likely that targeting the oxidationreduction imbalance, which admittedly has proven to be challenging, may be the more effective course, given the cascade of effects resulting from oxidant stress. Remodeling of the heart in ischemia, hypertrophy, and heart failure involves regionally disparate alterations of active membrane properties (i.e., ion channels, transporters, and pumps) as well as changes in cellular coupling. Disease-associated cellular coupling defects are in part due to changes in functional expression of connexins but, importantly, additional effects of remodeling of sodium current, changes in the extracellular matrix, and alteration of cell size and shape may undermine cellcell communication. The latter changes are often associated with disruptions in cellular structure including the cytoskeleton, having significant implications for trafficking of connexins and other proteins to the membrane. Finally, the role of alterations in the expression of other connexin isoforms in compensating or complicating defective $\mathrm{Cx} 43$ delivery to the plasma membrane should be considered.

It is difficult to overstate the importance of maintenance of normal gap junction function in the prevention of arrhythmias and maintenance of health. The work by Smyth et al. (9) provides important new molecular insights into the homeostatic regulation of $\mathrm{Cx} 43$ at gap junctions, providing yet another piece in the complex puzzle of regulation of cell-cell communication in the heart.

\section{Acknowledgments}

The author is supported by NIH grants RO1 HL050411, PO1 HL077180, and R33 HL087345.

Address correspondence to: Gordon F. Tomaselli, Division of Cardiology, Johns Hopkins University School of Medicine, 720 Rutland Ave., Ross 844, Baltimore, Maryland 21205, USA. Phone: (410) 955-2774; Fax: (410) 5022096; E-mail: gtomasel@jhmi.edu.

1. Maass K, Shibayama J, Chase SE, Willecke K, Delmar M. C-terminal truncation of connexin 43 changes number, size, and localization of cardiac gap junction plaques. Circ Res. 2007;101(12):1283-1291.

2. Beardslee MA, Laing JG, Beyer EC, Saffitz JE. Rapid turnover of connexin 43 in the adult rat heart. Circ Res. 1998;83(6):629-635.

3. Laird DW. Life cycle of connexins in health and disease. Biochem J. 2006;394(Pt 3):527-543.

4. Severs NJ, Bruce AF, Dupont E, Rothery S. Remodelling of gap junctions and connexin expression in diseased myocardium. Cardiovasc Res. 2008;80(1):9-19.

5. Musil LS, Goodenough DA. Multisubunit assembly of an integral plasma membrane channel protein, gap junction connexin 43 , occurs after exit from the ER. Cell. 1993;74(6):1065-1077.

6. Lauf U, Giepmans BN, Lopez P, Braconnot S, Chen SC, Falk MM. Dynamic trafficking and delivery of connexons to the plasma membrane and accretion to gap junctions in living cells. Proc Natl Acad Sci US A. 2002;99(16):10446-10451.

7. Giepmans BN, et al. Gap junction protein connexin-43 interacts directly with microtubules. Curr Biol. 2001;11(17):1364-1368.

8. Johnson RG, et al. Gap junctions assemble in the presence of cytoskeletal inhibitors, but enhanced assembly requires microtubules. Exp Cell Res. 2002;275(1):67-80.

9. Smyth JW, et al. Limited forward trafficking of connexin 43 reduces cell-cell coupling in stressed human and mouse myocardium. J Clin Invest. 2010;120(1):266-279.

10. Shaw RM, Fay AJ, Puthenveedu MA, von Zastrow M, Jan YN, Jan LY. Microtubule plus-endtracking proteins target gap junctions directly from the cell interior to adherens junctions. Cell. 2007;128(3):547-560.

11. Angst BD, et al. Dissociated spatial patterning of gap junctions and cell adhesion junctions during postnatal differentiation of ventricular myocardium. Circ Res. 1997;80(1):88-94.

12. Laing JG, Saffitz JE, Steinberg TH, Yamada KA. Diminished zonula occludens-1 expression in the failing human heart. Cardiovasc Pathol. 2007;16(3):159-164.

13. Kostin S. Zonula occludens- 1 and connexin 43 expression in the failing human heart. $J$ Cell Mol Med. 2007;11(4):892-895.

14. Barker RJ, Price RL, Gourdie RG. Increased association of ZO-1 with connexin 43 during remodeling of cardiac gap junctions. Circ Res. 2002;90(3):317-324.

15. Bruce AF, Rothery S, Dupont E, Severs NJ. Gap junction remodelling in human heart failure is associated with increased interaction of connexin 43 with ZO-1. Cardiovasc Res. 2008;77(4):757-765. 